\title{
Blockchain para registro, certificação e rastreabilidade de bens infungíveis
}

\author{
Blockchain for registration, certification and traceability of infungible property
}

Bruno Clemente Guingo ${ }^{1}$, Lucimar Cunha², Fabio Ribeiro Cerqueira ${ }^{3}$, Alberto Torres Angonese ${ }^{4}$, Eduardo Krempser ${ }^{5}$, Fabiano Saldanha Gomes de Oliveira6, Hugo Magalhães Queiroz ${ }^{7}$, Caio Rocha da Cunha ${ }^{8}$

\section{RESUMO}

Blockchain é uma tecnologia nova e emergente com grande potencial de revolucionar a indústria global com base na reconstrução das relações de confiança. Nos últimos anos a tecnologia blockchain vem sendo testada e aplicada para várias áreas e já existem muitos estudos de caso avaliando o uso prático em que a tecnologia blockchain foi aplicada. A indústria da arte e direitos autorais é mais uma área específica de possíveis aplicações da tecnologia blockchain. Este artigo apresenta uma Prova de Conceito ( $\mathrm{PoC})$, com estudo de caso, para validação, procedência e autenticidade de obras de arte e objetos de interesse artístico/arqueológico baseado em blockchain. O objetivo é verificar a capacidade da rede blockchain ser utilizada para realizar certificação, verificação e validação da autenticidade de obras de arte permitindo a geração de certificados permanentes de autenticidade para artistas.

Palavras-chave: Blockchain; Rastreabilidade; Rede Ethereum; Certificação Digital, Proveniência de obras de arte.

\section{ABSTRACT}

Blockchain is a new and emerging technology with great potential to revolutionize the global industry with the reconstruction of trust relationships. In recent years, blockchain technology has been tested and applied to several areas. There are already many case studies evaluating the practical use in which blockchain technology has been applied. The art and copyright industry is a specific area of possible uses of blockchain technology. This article presents a Proof of Concept (PoC) with case study for validation, provenance and authenticity of artworks and objects of artistic/archaeological interest based on blockchain. The objective is to verify the capacity of the blockchain network to enable certification, verification and validation of the authenticity of artworks, generating permanent certificates of authenticity for artists.

Keywords: Blockchain; Traceability; Ethereum Network; Digital Certification, Provenance of Artworks.

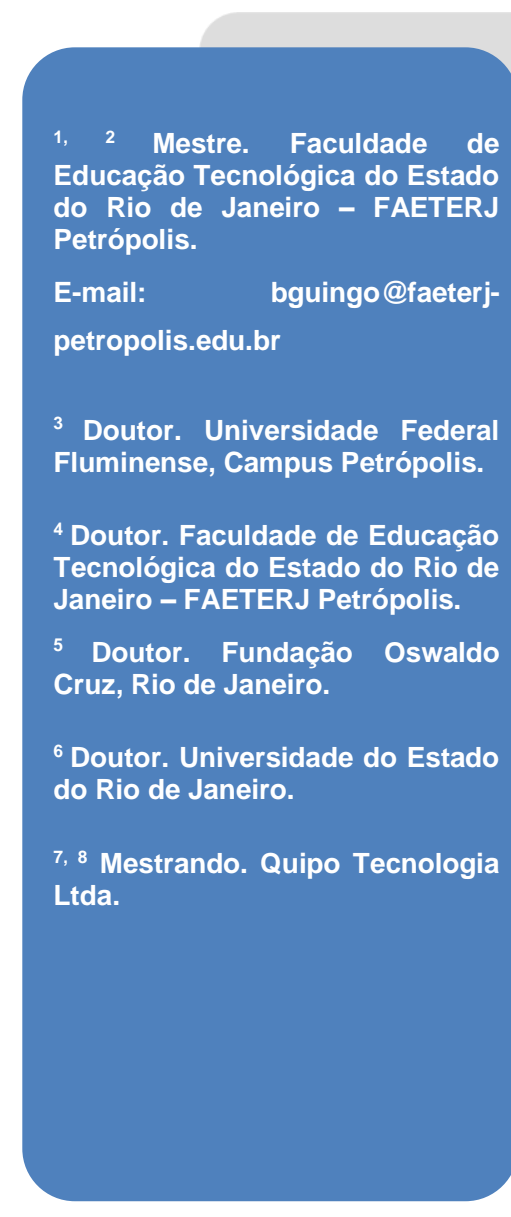




\section{INTRODUCĀO}

A tecnologia blockchain (cadeia de blocos) é um registro descentralizado e inviolável de dados e transações que desde a sua criação são registradas em blocos consecutivos, garantindo assim a permanência dos registros e, potencialmente, a facilidade no seu compartilhamento entre atores díspares em uma cadeia de valor.

Varella e Simeão (2018) conceituaram a cadeia de blocos da rede blockchain como sendo um livro eletrônico de registro distribuído, imutável e sem autoridade de controle que gera um protocolo de confiança calcado na abertura da informação sobre o negócio jurídico feito em ambiente virtual, numa rede computacional formada por pares equipotentes.

Também conhecida como tecnologia de "razão distribuída" ou "Livro Distribuído" foi projetada para apoiar a verificação e validação de dados e transações dentro de um ecossistema geralmente não confiável (ZHENG et al, 2018).

Atualmente, vivemos um período de profundas mudanças em relação à "confiança". As pessoas têm perdido a confiança nas instituições e no setor privado. Existe uma demanda imensa por novas formas de se estabelecer confiança, seja ela em governos, empresas e até mesmo nas relações pessoais.

Fato é que a blockchain é uma tecnologia que surgiu exatamente para gerar confiança de forma distribuída. O modelo de confiança é baseado primordialmente em sistemas centralizados ou descentralizados. Como um exemplo, pense que um cliente deseja saber quanto tem em sua conta bancária. Para isso, ele precisa perguntar à sua instituição financeira e confiar no que for dito por ela.

Com o uso de blockchain, no entanto, permite-se a criação de uma nova forma de confiança que não é centralizada (como no caso dos governos) nem descentralizada (como no caso do sistema financeiro global). É um modelo distribuído, e é por isso que esse sistema foi chamado de "confiança sem confiança", ou "trustless trust", no original em inglês.

Para fazer isso, a rede blockchain usa criptografia para assegurar a criação de um enorme banco de dados totalmente protegido contra adulteração (mesmo por seus operadores individuais). Ela pode ser descrita como um banco de dados, distribuído, capaz de produzir consenso e assegurar a integridade e unicidade das informações que nela são inseridas. 
Portanto, todas as ações realizadas na blockchain são gravadas de forma permanente, sem possibilidade de alteração. Dessa forma, um dado não pode ser alterado sem que se tenha um registro dessa alteração. Neste formato, a validação das transações do histórico de um produto torna-se altamente transparente e confiável para as partes envolvidas que, na cadeia de suprimentos, por exemplo, são os produtores, indústrias, distribuidores, varejistas e consumidor final.

Por isso, de forma bastante ilustrativa, pode-se pensar na tecnologia blockchain como um grande banco de dados, que armazena pedaços de informação interligados entre si, em blocos (daí o nome). No entanto, essa informação é armazenada de forma distribuída. Toda a rede "concorda" com aquela informação, gerando assim consenso sobre ela em toda parte. Essas informações são imutáveis. A probabilidade de adulteração da blockchain é praticamente zero. Além disso, a integridade e unicidade das informações são asseguradas em cada bloco.

Não sem razão, a tecnologia blockchain tem sido apontada como um sistema seguro, transparente e imutável de armazenamento distribuído de identidades e registros digitais; uma base para representar e rastrear digitalmente ativos mundiais como commodities, obras de arte, recursos naturais, resíduos etc., geralmente como tokens digitais, que são utilizados para representar um determinado ativo ou bem fungível ou infungível, passível de negociação. Qualquer indivíduo de posse de um token digital tem acesso ao item e pode rastreá-lo e negociá-lo digitalmente.

A cadeia de blocos literalmente projeta a confiabilidade para além das certificações digitais adotadas por um Estado, pois a utilização do programa que roda nesse ambiente gera um registro, que pode ter natureza contábil ou jurídica, que é imediata e automaticamente distribuído e gravado em todos os equipamentos de informática que já fizeram a operação ou farão, em escala planetária de milhões de computadores, de modo que quanto mais transações se fizer, mais segurança e confiança a blockchain proporciona ao usuário, pois mais difusa se torna a informação sobre o acordo celebrado e mais complexa fica a criptografia que incide sobre ele (NAKAMOTO, 2008).

A proposta de um sistema de registro e certificação de obras de arte com a tecnologia blockchain significa permitir aos artistas, museus, galeristas e demais participantes da cadeia da Arte a possibilidade de inserir informações na rede acerca de determinada obra, de maneira identificada e abertamente auditável, gerando confiança adicional ao processo de produção artístico, permitindo ainda a rastreabilidade da obra. 
Neste sentido, os principais objetivos deste artigo é conceituar a tecnologia blockchain e apresentar uma prova de conceito (PoC) para validar o uso da rede blockchain como efetiva para registro e certificação de obras de arte a partir do estudo de caso de registro das obras de arte do artista plástico Cocco Barçante na rede blockchain.

\section{MATERIAIS E METODOS}

O estudo de caso exploratório foi o método de pesquisa escolhido, por ser um método de pesquisa ampla sobre um assunto específico e pouco estudado, permitindo aprofundar o conhecimento sobre ele e, assim, oferecer subsídios para novas investigações sobre a mesma temática. Além disto, o estudo de caso é útil para investigar novos conceitos, bem como para verificar como são aplicados e utilizados na prática elementos de uma teoria (YIN, 2009).

Foi utilizado como estudo de caso o registro da coleção "Sentimentos do Rio", composta de 12 painéis produzidos pelo artista plástico Cocco Barçante. Tal coleção de autoria e propriedade do artista foi produzida ao longo dos últimos 10 anos e compõe o acervo do Museu do Artesanato do Estado do Rio de Janeiro, mas frequentemente é retirada do Museu para fins de exposição. Para registrar, certificar e validar a procedência da obra de artista vivo foi necessário o desenvolvimento de um sistema que permitisse e garantisse a integridade do registro da produção artística na rede blockchain.

A metodologia empregada na elaboração desse sistema se deu em doze fases distintas e visam organizar e sistematizar todo o trabalho realizado pela equipe de desenvolvimento.

$\checkmark$ Fase 1 - Levantamento de Requisitos: Listar todos os requisitos funcionais para construção de uma solução para registro de dados e transações na rede blockchain.

$\checkmark$ Fase 2 - Estudo da Viabilidade de Modelo: Definir os melhores modelos para implementação do Registro da Obra.

$\checkmark$ Fase 3 - Definição do Modelo: Escolher qual modelo melhor se enquadra para funcionalidade definida.

$\checkmark$ Fase 4 - Concepção, Especificação e Design da Interface: Definir o escopo da interface do sistema de registro de obra na rede blockchain.

$\checkmark$ Fase 5 - Prototipação e Design da Arquitetura: Definir o protótipo e o design da arquitetura do sistema. 
$\checkmark$ Fase 6 - Definição do Banco de Dados: Escolher o banco de dados e sua modelagem.

$\checkmark$ Fase 7 - Desenvolvimento das Rotinas e Algoritmos: Elaborar as rotinas e algoritmos necessários para funcionamento do sistema.

$\checkmark$ Fase 8 - Desenvolvimento das Rotinas de Processamento de Imagens: Elaborar as rotinas e algoritmos de processamento de imagens.

$\checkmark$ Fase 9 - Implementação das Funcionalidades: Implementar as funcionalidades necessárias.

$\checkmark$ Fase 10 - Realização de Testes das Rotinas de Processamento de Imagens: Efetuar os testes para verificação das Rotinas de Processamento de Imagens.

$\checkmark$ Fase 11 - Realização de Testes de Acordo com os Requisitos: Efetuar os testes para verificação da funcionalidade dos Requisitos do Sistema.

$\checkmark$ Fase 12 - Disponibilizar o sistema para uso do artista para fins de efetivação de registro de obra de arte na rede blockchain.

O estudo de caso ora apresentado, baseado no registro da obra "Harmonia" é considerado adequado para a prova de conceito e teste do sistema de registro e avaliação da capacidade da rede blockchain funcionar como certificadora segura de obras de arte, uma vez que envolve todos os problemas de informação e registro supostamente abordados pela tecnologia blockchain.

A base de dados inicial de teste da plataforma e realização do estudo de caso foi constituída por 12 painéis de autoria do artista plástico Cocco Barçante, na figura 1 é apresentada de forma exemplificativa a ficha técnica registrada na Rede Ethereum da Obra de Arte "Harmonia". 


\section{й \\ COCCO BARÇANTE}

\section{Cocco Barosiste}

\section{HARMONIA}

TÉCNICA/MATERIAL

Pintura e Bordado sobre Lona/Tecido

DIMENSÕES ( $L \times A)$

Painel - 2,50 X 2,00 Metros

DATA DE PRODUÇÃO 2010

PROPRIETÁRIO ATUAL

Acervo do Museu do Artesanato do Estado do Rio de Janeiro.

URL

http://146.134.225.24/batch/226/

NOTA DE REGISTRO

Harmonia - painel criado para representar 0

momento das pacificações nas comunidades do Rio de Janeiro. Sob encomenda, com uma linguagem personalizada.
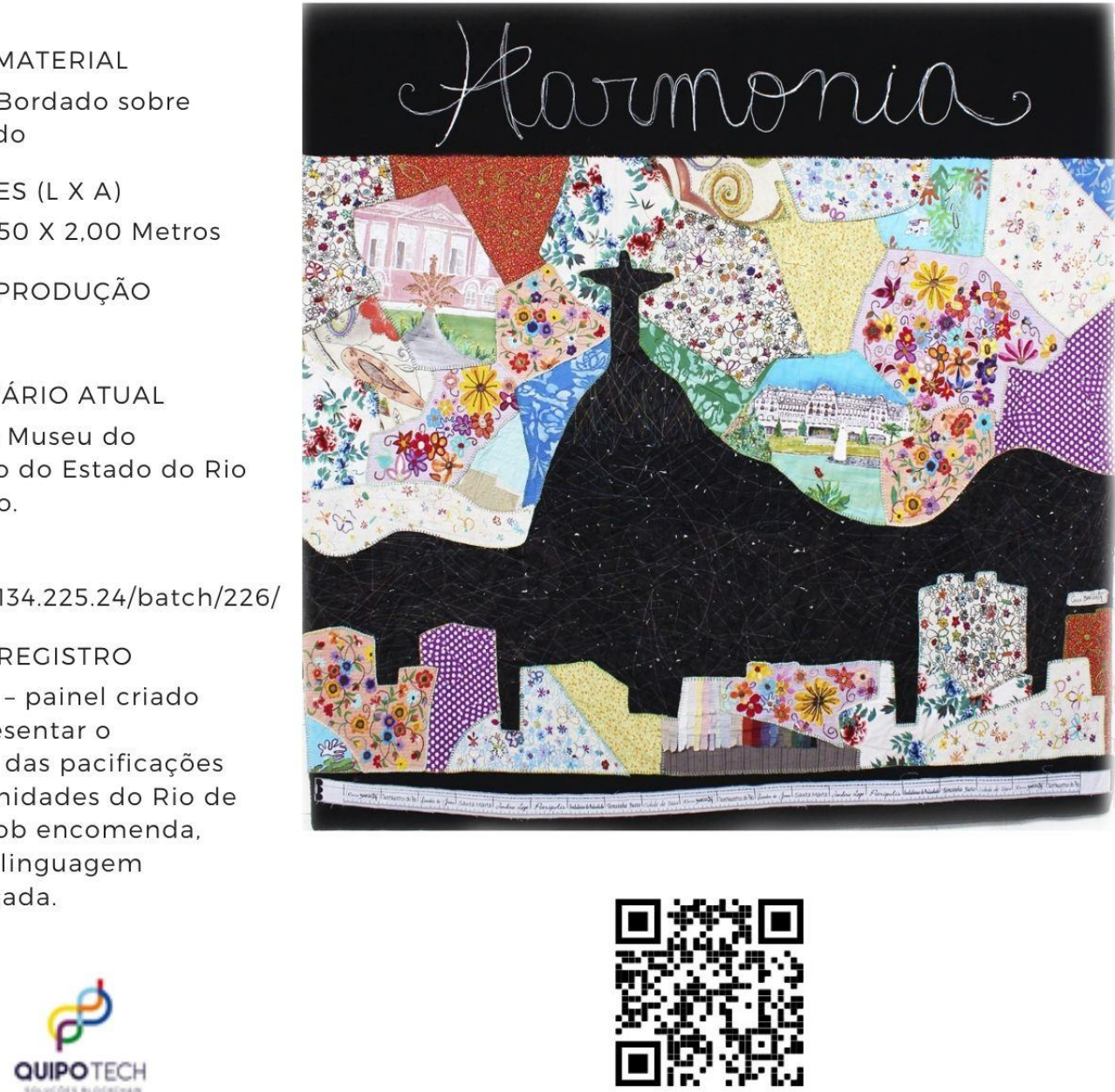

(B) VERIFICADO POR QUIPOART

Figura 1. Ficha Técnica da Obra Harmonia registrada em blockchain (Rede Ethereum). Fonte: Autores, 2020. 
A obra Harmonia foi registrada na Rede Ethereum com o seguinte código Hash associadoํㅜ 0xC896DFł31967FedA8A494F194DE77adab8B571B2 que pode ser verificado a partir de http://146.134.225.24/batch/226/.

\section{RESULTADOS}

Com o desenvolvimento da prova de conceito foi possível realizar com sucesso o registro de 12 painéis artísticos de autoria do artista plástico Cocco Barçante. Foram registradas as obras, suas fichas técnicas, documentação de atributos das obras, imagens, dados do autor/artista, data de criação. Tal registro possibilitou ao artista um certificado digital tokenizado para cada uma das obras de arte registradas. O certificado contém informações verificáveis sobre a peça que podem ser facilmente transferidos ao longo da cadeia criando uma segura rede de rastreabilidade.

Com este estudo de caso foi concluído que como os tokens podem ser usados para representar informações e ativos, a plataforma baseada em blockchain pode permitir que grandes grupos de pessoas compartilhem dados confiáveis sem depender de uma autoridade central. Isso também pode alavancar ainda mais os mercados de arte online de forma efetivamente mais segura e confiável, pois mercados em que credenciais para obras de arte, artistas, compradores e vendedores podem ser facilmente autenticadas e verificadas tendem a se fortalecer no futuro.

As experiências nesta fase, além de permitir o desenvolvimento e validação das ferramentas de registro em blockchain, possibilitou o aprimoramento de conceitos de usabilidade e viabilidade das soluções, bem como analisar o conjunto de dados (e interações dos usuários) recolhido durante o processo de registro da obra, que foi realizada diretamente pelo artista.

Durante esse processo, foi possível também identificar o potencial didático da ferramenta. Além de evidenciar a preocupação pelo aprimoramento das melhores práticas em cada etapa de registro, mostrando a possibilidade de compartilhamento de informações "anonimizadas" de processos que podem beneficiar toda comunidade.

O projeto foi adequadamente desenvolvido para uma versão Web e Móvel, visando ao acesso rápido, prático e inteligente. Mais especificamente, o núcleo do desenvolvimento é formado por um conjunto de APIs (Application Programming Interface) principalmente desenvolvida em linguagem de programação Python e capaz de fornecer e

\footnotetext{
${ }^{1}$ https://etherscan.io/address/0xC896DFf31967FedA8A494F194DE77adab8B571B2
} 
adaptar inúmeros serviços para diferentes interfaces com o usuário e funcionalidades finais. Isso significa dizer que inúmeras formas de acesso do usuário final podem ser idealizadas sem a alteração do núcleo do seu desenvolvimento e sem afetar a segurança das informações tratadas e armazenadas. Exemplo dessa flexibilidade é permitir diferentes formatos de acesso Web, smartphones e até mesmo loT (Internet das Coisas).

A rede blockchain escolhida para registro da obra foi a Ethereum, por ser uma plataforma global de código aberto para aplicativos descentralizados, sendo atualmente a principal rede Blockchain programável no mundo. Sendo global e aberta, reafirma sua garantia da transparência, confiabilidade dos dados e escalabilidade.

\section{DISCUSSÃO}

O uso da tecnologia de blockchain inserida dentro de aplicativos ou sistemas web traz diversos benefícios aos usuários artistas, uma vez que permite de forma simplificada e econômica, o registro e comprovação de paternidade de uma obra, garantindo assim a propriedade intelectual.

As iniciativas baseadas em aplicações de blockchain e smart contracts devolverão aos artistas o controle de suas obras. Assim, eles poderão mostrar ou vender seus trabalhos para potenciais compradores em um ambiente online seguro e a preços rentáveis. É o que faz a Maecenas Fine Art, pioneira nos leilões de arte via blockchain. Nessa plataforma, os vendedores fazem um inventário de suas obras, e os investidores podem comprá-las por fragmentos, tornando o custo da operação mais baixo. Sem intermediários e com a transparência, rastreabilidade e rapidez que o blockchain possibilita (MAECENAS, 2020).

O blockchain também pode ser aplicado a inovações na área de direitos autorais. Graças a essa tecnologia, podemos armazenar de forma permanente a propriedade intelectual de um trabalho na nuvem e administrá-la de onde e quando quisermos. Isso melhora a transparência e garante os direitos autorais em um momento delicado para a evolução da arte na internet: em 2017, foram feitas 300 bilhões de visitas a sites de conteúdo pirata no mundo, 1,6\% a mais em relação a 2016, de acordo com estudo da empresa Muso, sediada em Londres, sobre pirataria online (MUSO, 2020).

A Kodak e a Baidu são duas empresas com iniciativas blockchain para proteger e gerenciar a propriedade intelectual das fotografias que circulam na rede. A primeira por 
meio de uma criptomoeda única para comprar imagens dentro de sua própria plataforma (KODAK, 2020); e a segunda usa um app onde as informações de copyright ficam armazenadas no blockchain (BAIDU, 2020).

Outra aplicação da tecnologia blockchain é melhorar a rastreabilidade das obras de arte. Como se trata de uma tecnologia criptográfica complexa, podemos registrar o percurso completo de um quadro, escultura ou qualquer objeto valioso, desde a criação até seu destino final. Com a tecnologia blockchain são armazenadas informações de, entre outras coisas, registros, vendas, estudos, análises, certificações e avaliações de uma obra. Assim, é possível reduzir o tráfico ilícito de bens culturais e também resolver o problema das falsificações.

\section{CONSIDERAÇÕES FINAIS}

Neste artigo é apresentado uma Prova de Conceito para avaliar a pertinência do uso da rede blockchain para registro, certificação, validação e rastreabilidade de Obras de Arte.

A utilização da tecnologia blockchain para o desenvolvimento das funcionalidades sugeridas e testadas na prova de conceito atende de forma satisfatória a todas as propostas de concepção de uma infraestrutura baseada em tecnologia blockchain para certificados e proveniência artística para obras de arte e objetos de interesse artístico e arqueológico. O registro de autoria de obra de arte em blockchain na rede Ethereum, é claramente possível e efetivo, pois além do registro pode-se efetuar a certificação e rastreabilidade digital da obra com informações verificáveis sobre a peça, permitindo a verificação da autenticidade e procedência de obras e objetos de interesse arqueológico. Todos esses fatores combinados dão aos usuários uma confiança de uso e garantia da veracidade das informações sobre as obras de arte.

A implementação experimental proposta a partir do registro dos 12 painéis "Sentimentos do Rio" de autoria do artista plástico Cocco Barçante, com foco na certificação das obras via rede blockchain, nos forneceu resultados satisfatórios, demonstrando que a rede blockchain fornece uma solução completa, segura e econômica, de simples utilização por parte do usuário artista e agrega valor a cada uma das obras registradas, com a geração de sua respectiva ficha técnica, documentação referente aos atributos da obra, imagem, dados do artista, do proprietário atual, trazendo 
transparência e garantindo de forma irreversível a paternidade da criação com emissão de certificado digital tokenizado.

Por fim, considerando a avaliação da rede blockchain como aplicável ao registro seguro de obras de arte, entendemos que a solução testada foi projetada apenas para realizar certificações digitais, podendo a partir de uma infraestrutura expansível, no futuro, se incorporar outras facilidades oferecidas pela tecnologia blockchain, como os contratos inteligentes para a efetivação da transferência de propriedade de uma obra de arte, bem como a integração de soluções inteligentes de loT, como monitoramento, confirmação da autenticidade da obra por processamento de imagem, georreferenciamento e registro biológico, em conformidade com as demandas de cada artista, museu ou colecionador.

\section{REFERÉNCIAS}

ARTORY. 25 Nov 2019. Portal. Become a collector: register your artwork. Disponível em: <https://www.artory.com/>. Acesso em: 04 de agosto de 2020.

BAIDU. 2020. Portal. Baidu launches copyright protection blockchain. Disponível em: $<$ https://ledger-insights.com/baidu-copyright-protection-blockchain/>. Acesso em: 07 de julho de 2020.

\section{FIGURELLI, R. BLOCKCHAIN: Uma análise estratégica para humanos e robôs.} Trajecta. Edição do Kindle, 2017.

IBERDROLA. 2020. Portal. O 'blockchain' revolucionará a indústria da arte. Disponível em: <https://www.iberdrola.com/cultural/aplicacoes-blockchain-na-arte>. Acesso em: 06 de agosto de 2020.

KODAK. 07 Jun 2020. Portal. Kodak launches a blockchain-enabled document management system. Disponível em: <https://www.coindesk.com/kodak-launched-ablockchain-enabled-document-management-system>. Acesso em: 26 de junho de 2020.

LAGO, L. Blockchain: confiança através de algoritmos. Boletim, vol. 2, n. 4. São Paulo: CEST/USP, 2017.

MAECENAS. The art investment platform. Disponível em: <https://www.maecenas.co/>. Acesso em: 06 de abril de 2020.

MUSO. Publishing under threat as demand for illegal content rises over 2018. Disponível em: <https://www.muso.com/magazine/publishing-under-threat>. Acesso em: 10 de abril de 2020.

NAKAMOTO, S. Bitcoin: a peer-to-peer eletronic cash system. Disponível em: <https://git.dhimmel.com/bitcoin-whitepaper/>. Acesso em: 7 nov. 2017.

TAPSCOTT, D., TAPSCOTT, A. Blockchain revolution. Editora Senai-SP, 2017. 
VARELLA, D., SIMEÃO, A. Equipotência libertária do ciberespaço e a regulação transnacional da cadeia de blocos (blockchain) R. Dir. Gar. Fund., Vitória, v. 19, n. 3, p. 99-126, set./dez. 2018.

YIN, R. K. Case study research, design and methods (applied social research methods). Thousand Oaks. California: Sage Publications, 2009.

ZHENG, Z., XIE, S., DAI, H., CHEN, X., WANG, H. Blockchain challenges and opportunities: a survey. International Journal of Web and Grid Services, vol. 14, no.4, pp.352-375, 2018. 